\title{
Behavioral Disorder
}

National Cancer Institute

\section{Source}

National Cancer Institute. Behavioral Disorder. NCI Thesaurus. Code C35470.

A specific behavioral problem that occurs in persistent patterns and characteristic

clusters and that causes clinically significant impairment. 